\title{
The Focal Heat Flux Distribution Measurement of High-times Concentrating Directional Transmission System
}

\section{S. L. Wu, H. P. Tan, X. Chen, and X. L. Xia}

Key Laboratory of Aerospace Thermophysics of MIIT, Harbin Institute of Technology, Harbin 15001, China

\section{Abstract}

The concentrating performance of solar energy plays an important role in its application at high temperature. Recently, High-times Concentrating Directional Transmission System (CDTS) was built in the authors' laboratory. To evaluate the focal flux distribution of a solar concentrator, a kind of measurement system was established, in which an infrared camera and several thermocouples along with a

Corresponding Author:

X. L. Xia

xiaxl@hit.edu.cn

Received: 14 September 2018

Accepted: 1 October 2018

Published: 14 October 2018

Publishing services provided by Knowledge E

(c) S. L. Wu et al. This article is distributed under the terms of the Creative Commons

Attribution License, which permits unrestricted use and redistribution provided that the original author and source are credited.

Selection and Peer-review under the responsibility of the ASRTU Conference Committee.

\section{G OPEN ACCESS} heat flow meter were employed, and the clear heat flux distribution of focal spot was obtained. The skew ray tracing principle was used to simulate the process of solar energy transmission and compared with the test results.

Keywords: measurement and instrument, CDTS, heat flux distribution, skew ray tracing principle

\section{Introduction}

Solar energy is an abundant and renewable energy source. The development and utilization of solar energy resources is of great significance for energy conservation, emission reduction, and improvement of the ecological environment. The research on space solar energy concentrating and transmission technology provides the necessary technical conditions for the establishment of concentrating solar space power station [1]. In order to effectively utilize the low-density radiant energy of solar energy, it is necessary to use optical aggregation technology to increase the concentrating ratio and obtain high-energy radiant energy. At present, solar energy primary and secondary concentrating technologies have been mastered internationally. For space solar concentrating systems, obtaining effective concentrating ratio and concentration 
of solar current density plays a crucial role in improving conversion efficiency and reducing thermal control technology.

The focal heat flux distribution is a key factor in evaluating the concentrating effect of the concentrator and plays a decisive role in the design, installation, and efficiency analysis of the concentrating collector system and the concentrating photovoltaic system. At present, the methods for focal plane measurement of large concentrators mainly include direct measurement method and indirect one.

The direct measurement method consists in installing a heat flux density sensor at a finite discrete point position at the focal plane of the concentrating system and acquiring the value from it. Jesus Ballestrin et al. [2-4] designed and manufactured a direct measurement system for focal plane energy flow density distribution for solar tower photothermal power plants. The advantages of this experimental measurement system are short response time, accurate system measurement, and absence of water cooling system, while the disadvantage is low measurement resolution.

The indirect method measures the energy density field by placing a Lambertian target at the focal plane of the concentrating system, photographing it with a CCD camera or a digital imaging radiometer, and then establishing a functional relationship between the image and the energy flow, and obtaining the energy density by inversion field. Johnston et al. $[5,6]$ used an indirect measurement method to study the focal plane energy density field distribution of a dish-type concentrating system in a WhiteCliffs solar thermal power plant. The distribution of the energy density field of the focal plane of the dish concentrator was measured by CCD camera and Lambertian reflection surface, and compared with the simulation results. The indirect measurement method has the advantages of high resolution, short measurement time, fast response, etc., but the data obtained are the pixel gray value of the image, the real energy density value cannot be obtained, and the camera is introduced and concentrated. The reflection characteristics increase the measurement error.

In this article, a clear infrared image of concentrated solar energy distribution of High-times Concentrating Directional Transmission System (CDTS) is acquired using infrared thermal imager, heat flow meter and thermocouple, using direct and indirect measurement methods. The skew ray tracing principle was used to simulate the transmission process. 


\section{Methods}

\subsection{Model description}

The High-times Concentrating Directional Transmission System (CDTS) uses a single plane heliostat for real-time tracking to reflect incident solar rays, and then the reflected parallel light is concentrated by the secondary parabolic mirror to the focal spot, so that the receiving surface obtains a high flux distribution. The advantage of this method is that the tracking and adjustment of the primary mirror is easy, and it is not necessary to adjust the movement of the secondary mirror and the heat absorber, which is convenient to arrange the relevant experimental systems, and the Sketch of ray transmission is shown in Figure 1.

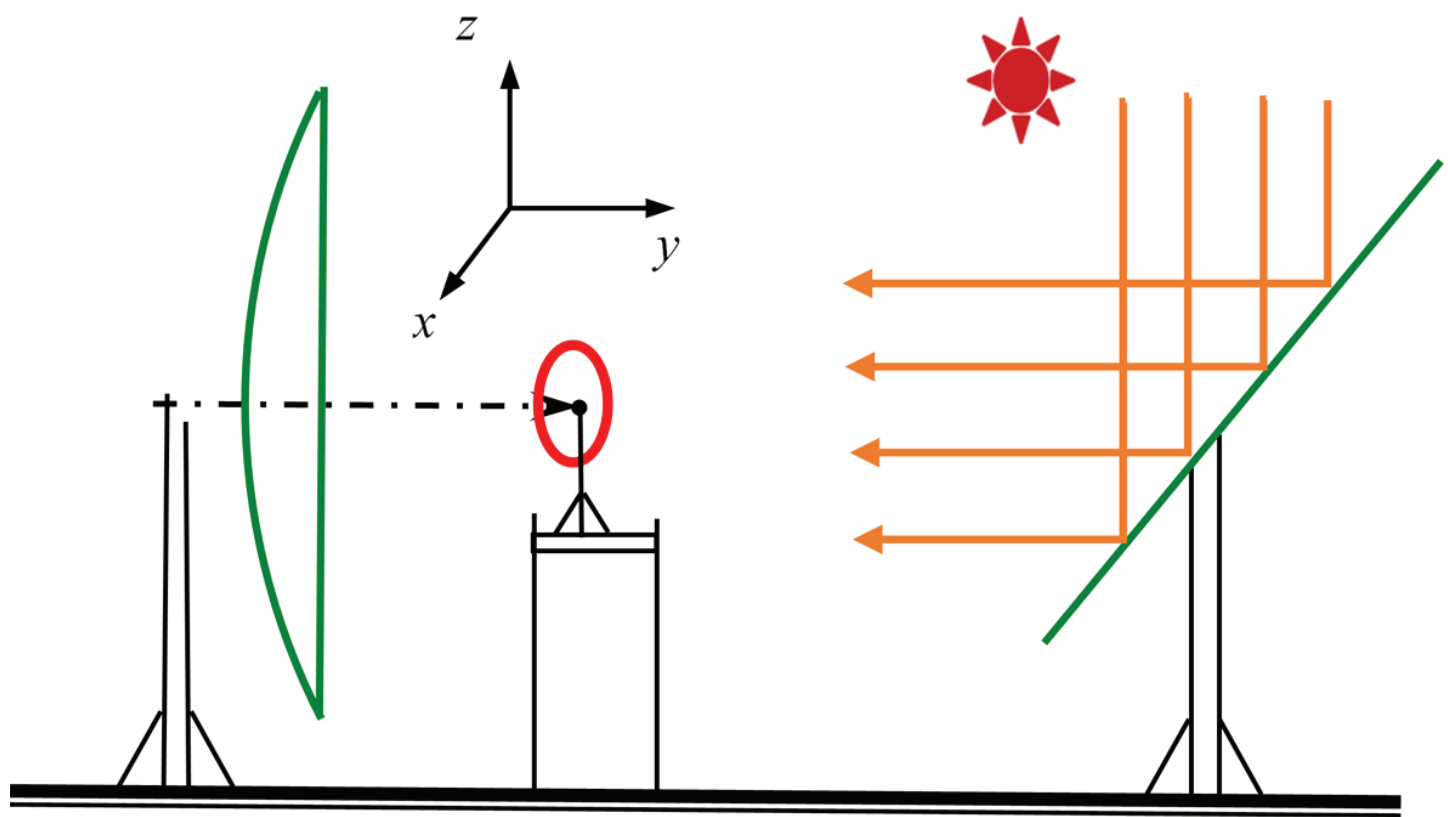

Figure 1: Schematic image of ray transmission.

The High-times Concentrating Directional Transmission System (CDTS) as shown in Figure 2. The layout is selected from north to south. There are several facilities from north and south: heliostats, heat absorber, and dish condensers. The heliostat is composed of $9 \times 7$ plane unit mirrors, the size of each unit mirror is $1.218 \times 0.913$ $\mathrm{m}^{2}$, and the whole mirror of the heliostat is flat, the overall size is $8.706 \times 8.457 \mathrm{~m}^{2}$. The concentrated mirror is composed of 19×20 unit plane mirrors deformed by curved surface. The size of each unit plane mirror is $0.45 \times 0.45 \mathrm{~m}^{2}$. The concentrating mirror has a surface with a rotating paraboloid, and its focal length is $6.92 \mathrm{~m}$. Finally, the geometric center points of the heliostat, the dish mirror, and the opening plane of 
the heat absorber are all on the same horizontal line, and the height of the center point is $5.5 \mathrm{~m}$. The reflectivity of heliostats and dish concentrators during the numerical simulation is provided by the factory at 0.8 .

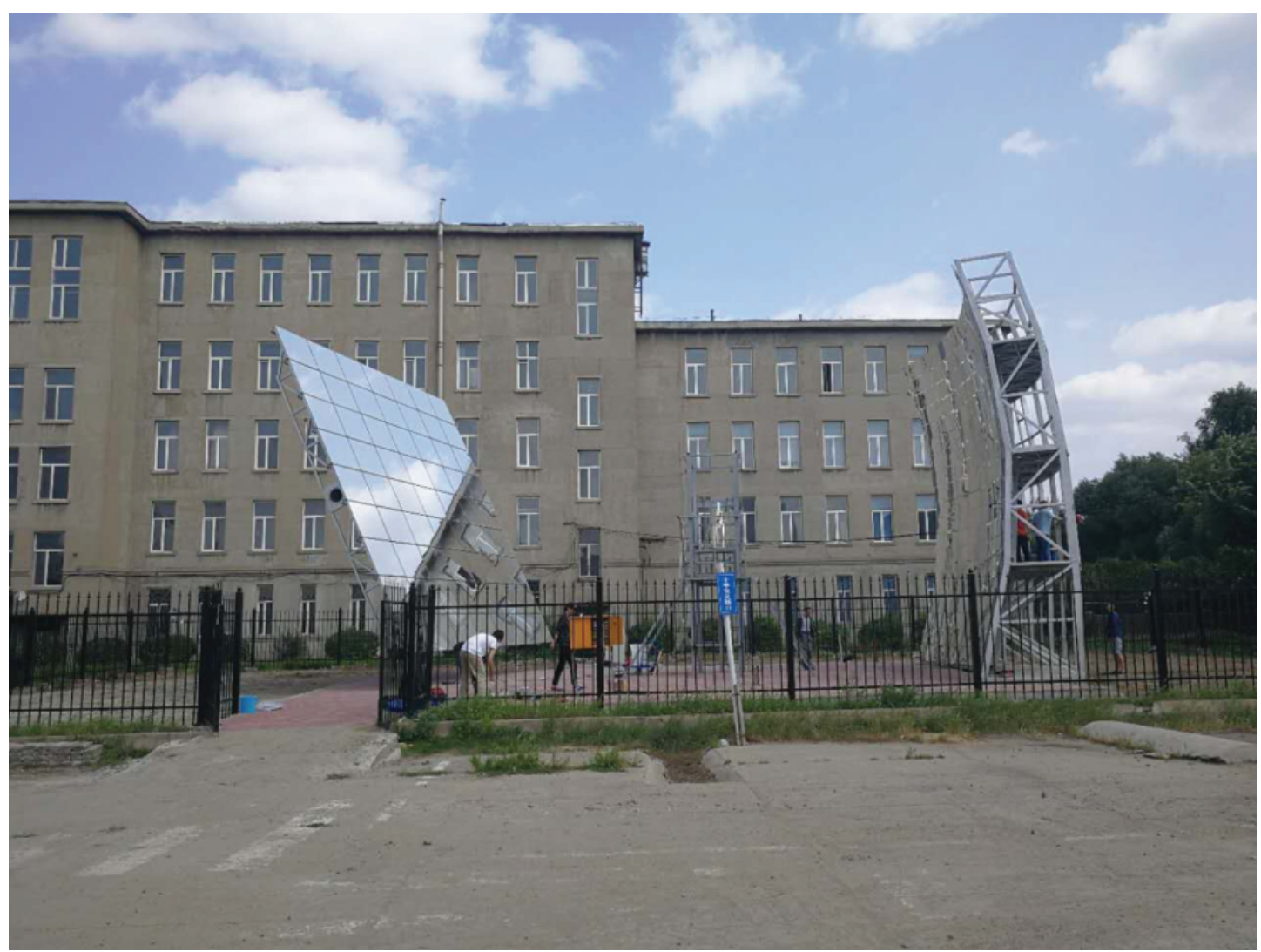

Figure 2: CDTS system.

\subsection{Simulation methods}

The skew ray tracing principle is used to model the system. The optical performance of the tracking system is simulated by the method of checkerboard sequence.

There are three types of light propagation in an optical system: paraxial ray, meridional ray, and skew ray [7]. The traditional optical system design principle is based on parallel incident light. The incident light is always kept in a plane with the optical axis of the system. For example, for the lens and parabolic reflecting optical system, all the incident light rays can be concentrated at the focus. In contrast, skew light means the general light. Vector analysis of this type of light cannot rely on simple optical theory, according to the basic vector theory (such as the law of reflection/refraction).

In the field of computer graphics, in order to realize flexible transformations such as displacement, rotation, and scaling between graphics, a homogeneous coordinate 
matrix representation is often introduced [8]. The homogeneous coordinate system uses $n+1$ components to represent $n$-dimensional coordinates. For example, in a threedimensional Cartesian coordinate system, the $3 \times 3$ matrix expression is expanded to a $4 \times 4$ matrix, and new data are used for translation transformation. Realize the integrated transformation of matrix multiplication and translation. By using the representation method of the homogeneous coordinate matrix, any transformation matrix can be obtained by matrix multiplication, which improves the computational efficiency.

In the $4 \times 4 \mathrm{D}-\mathrm{H}$ coordinate system, the axisymmetric surface can always be obtained by rotating a single curve around its own axis of symmetry. Therefore, the local coordinate arbitrary surface with the $y$ axis as the axis of symmetry can be expressed as:

$$
F_{\mathrm{w}}=\operatorname{Rot}\left(y, \alpha_{w}\right) S_{w}=\left[\begin{array}{lll}
x\left(\beta_{w}\right) \cos \alpha_{w} & y\left(\beta_{w}\right)-x\left(\beta_{w}\right) \sin \alpha_{w} & 1
\end{array}\right]^{T}
$$

For a flat surface, the boundary line can be expressed as:

$$
S_{\mathrm{w}, \mathrm{flat}}=\left[\begin{array}{llll}
\beta_{\mathrm{w}} & 0 & 0 & 1
\end{array}\right]^{\top}
$$

\subsubsection{Skew ray tracing principle}

The heliostat with the two-axis tracking system uses the mirror reflection principle to ensure that the solar imaging is always on the optical axis of the dish condenser, so that the incident light of the secondary mirror is parallel to the optical axis. Under the local coordinates and viewing angle of a certain fixed point on the ground, the sun tracking incident angle can be determined by the elevation angle $\alpha$ and the circumferential angle $A_{z}$.

$\mathrm{v}_{\mathrm{s}}$ is a reflected light vector, $\mathrm{v}_{t}$ is an incident ray vector, $\theta$ is the equal angle of Incident/Reflection vector. $K$ is the direction of the symmetry axis that rotates the incident-reflective normal vector.

$$
\mathrm{v}_{\mathrm{s}}=\left[\begin{array}{llll}
\cos \alpha \sin \mathrm{A}_{\mathrm{z}} & \cos \alpha \cos \mathrm{A}_{\mathrm{z}} & \sin \alpha & 0
\end{array}\right]^{\top}
$$

According to dish concentrator, reflected light vectorv $\mathrm{v}_{\mathrm{t}}$ :

$$
\mathrm{v}_{\mathrm{t}}=\left[\begin{array}{llll}
0 & -1 & 0 & 0
\end{array}\right]^{\top}
$$

Normal vector of CDTS system:

$$
n_{1}=\operatorname{Rot}\left(\mathrm{k}, \theta_{1}\right)\left(-\mathrm{v}_{\mathrm{s}}\right) \text {, }
$$


where $\theta_{1}$ is the equal angle of Incident/Reflection vector:

$$
\theta_{1}=\frac{1}{2} \cos ^{-1}\left(-\mathrm{v}_{\mathrm{s}} \cdot \mathrm{v}_{\mathrm{t}}\right)
$$

$\mathrm{k}$ is the direction of the symmetry axis that rotates the incident-reflective normal vector. The product of the incident and reflected vectors:

$$
k=\mathrm{v}_{\mathrm{s}} \times \mathrm{v}_{\mathrm{t}}
$$

$\operatorname{Rot}\left(\mathrm{k}, \theta_{1}\right)$ is a normal vector rotation matrix based on the local rotating coordinate system, it is positive for counterclockwise direction, otherwise it is clockwise.

$$
\operatorname{Rot}\left(\mathrm{k}, \theta_{1}\right)=\left[\begin{array}{cccc}
k_{x} k_{x}\left(1-\cos \theta_{1}\right)+\cos \theta_{1} & k_{y} k_{x}\left(1-\cos \theta_{1}\right)-k_{z} \cos \theta_{1} & k_{z} k_{x}\left(1-\cos \theta_{1}\right)+k_{y} \sin \theta_{1} & 0 \\
k_{x} k_{y}\left(1-\cos \theta_{1}\right)+k_{z} \sin \theta_{1} & k_{y} k_{y}\left(1-\cos \theta_{1}\right)+\cos \theta_{1} & k_{z} k_{y}\left(1-\cos \theta_{1}\right)-k_{x} \sin \theta_{1} & 0 \\
k_{x} k_{z}\left(1-\cos \theta_{1}\right)-k_{y} \sin \theta_{1} & k_{y} k_{z}\left(1-\cos \theta_{1}\right)+k_{x} \sin \theta_{1} & k_{z} k_{z}\left(1-\cos \theta_{1}\right)+\cos \theta_{1} & 0 \\
0 & 0 & 0 & 1
\end{array}\right]
$$

\subsubsection{Intersection solution method in sequence tracing process}

As shown in the Figure 3, suppose that in the coordinate system, the start point $P_{\mathrm{s}}=$ $\left[\begin{array}{llll}P_{s, x} & P_{s, y} & P_{s, z} & 1\end{array}\right]^{T}$ is extended in a certain direction $\mathrm{v}=\left[\begin{array}{llll}\mathrm{v}_{\mathrm{s}, x} & \mathrm{v}_{s, y} & \mathrm{v}_{s, z} & 0\end{array}\right]^{T}$. In the vector direction, the transmitted oblique light hits a certain surface and intersects at the point $P=\left[\begin{array}{llll}P_{x} & P_{y} & P_{z} & 1\end{array}\right]^{T}, P$ can be expressed by the following formula:

$$
P=\left[P_{s, x}+\lambda v_{s, x} P_{s, y}+\lambda v_{s, y} P_{s, z}+\lambda v_{s, z} 1\right]^{T}
$$

$\lambda$ is the scalar length of the vector line $\overrightarrow{P_{s} P}$.

Therefore, if you can get the $\lambda$ value, you can know the position of the boundary point $P .{ }^{\mathrm{w}} P={ }^{w} A_{0} P$ can be solved separately with the formulas (10) and (11) to obtain the scalar values of the corresponding dish concentrator and plane receiving surface.

$$
\lambda=\frac{-\left(I_{y} P_{\mathrm{s}, x}+J_{y} P_{\mathrm{s}, y}+K_{y} P_{\mathrm{s}, z}+t_{y}\right)}{I_{y} L_{\mathrm{s}, x}+J_{y} L_{\mathrm{s}, y}+K_{y} L_{\mathrm{s}, z}}
$$

When next intersection is a paraboloid, $\lambda$ can be solved by:

$$
\begin{aligned}
& {\left[\left(I_{x} \mathrm{v}_{s, x}+J_{x} \mathrm{v}_{s, y}+K_{x} \mathrm{v}_{s, z}\right)^{2}+\left(I_{y} \mathrm{v}_{s, x}+J_{y} \mathrm{v}_{s, y}+K_{y} \mathrm{v}_{s, z}\right)^{2}\right] \lambda^{2}+} \\
& {\left[2\left(I_{x}+I_{y}-2 f I_{z}\right) v_{s, x}+2\left(J_{x}+J_{y}-2 f J_{z}\right) v_{s, y}+2\left(K_{x}+K_{y}-2 f K_{z}\right) v_{s, z}\right] \lambda+} \\
& \left(I_{x} P_{s, x}+J_{x} P_{s, y}+K_{x} P_{s, z}+t_{x}\right)^{2}+\left(I_{y} P_{s, x}+J_{y} P_{s, y}+K_{y} P_{s, z}+t_{y}\right)^{2} \\
& -4 f\left(I_{z} P_{s, x}+J_{z} P_{s, y}+K_{z} P_{s, z}+t_{z}\right)=0
\end{aligned}
$$




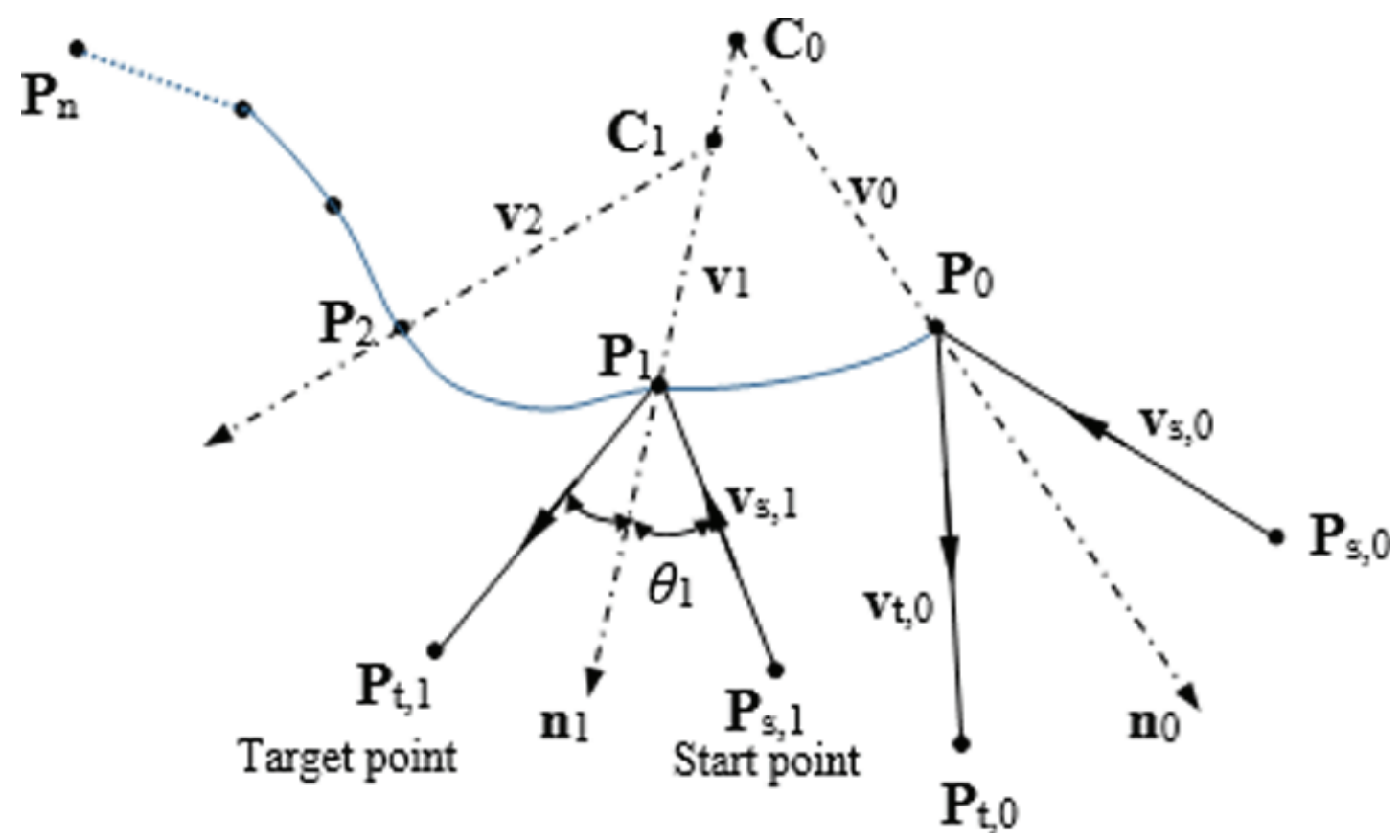

Figure 3: Generating freeform curve using 2D-CGCM.

\subsection{Experimental methods}

In this article, the infrared thermal imager was used to obtain the infrared radiation energy flow distribution of the surface, and the actual concentrated energy flow distribution of the focal spot was calculated by combining the measurement data of the high energy density detector. The system consists of a flow density detector (heat flow meter), a water-cooled Lambertian target, thermocouples, a device mount, a watercooled box, a controller, a heat flow data collector, and an infrared camera (Figure 4). The highest energy density value of the spot center was obtained; since the energy of the concentrated spot was high, the heat flow meter and the Lambert target need to be cooled, so that water cooling equipment was provided; the overall distribution of the focal spot was obtained using an infrared thermal imager, and the distribution data were combined. The heat flow meter measurement resulted in the actual aggregated energy flow distribution, and was corrected and tested by thermocouples [9]. The radiant energy entering the thermal imager includes reflection of concentrated solar radiant energy, Lambertian surface's own infrared radiant energy, and environmental radiant energy.

The experiment of CDTS system was performed on a platform. The radiant energy flow detected by the infrared camera includes the solar radiant energy reflected by the target surface, the radiant energy emitted by itself, and the amount of environmental 
(a)

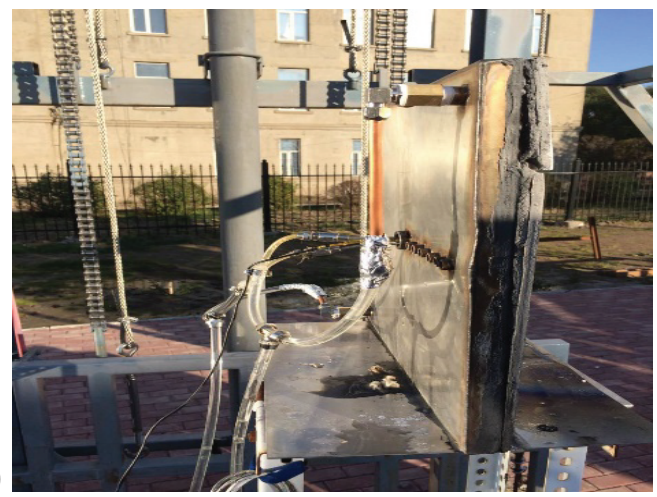

(b)

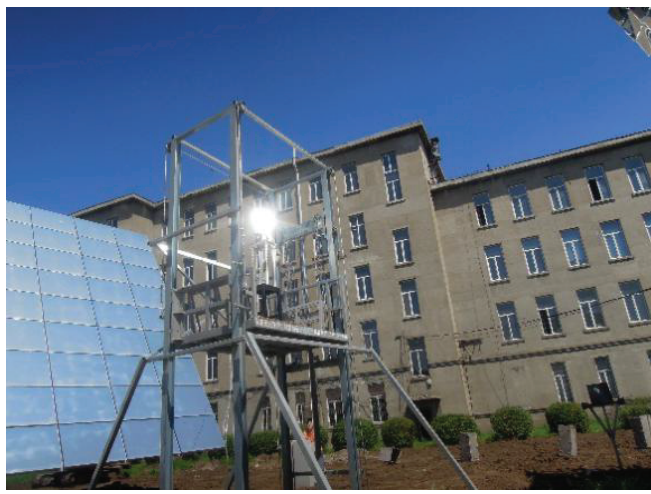

Figure 4: Experimental study of concentrated heat flux distribution.

radiation. After obtaining the infrared energy flow distribution, the actual flux flow distribution of the receiving surface can be solved by the formula (1):

$$
C_{\mathrm{NE}}=\frac{\epsilon_{\mathrm{lr}} \sigma T_{\mathrm{lL}}^{n}-\int_{7.5 \mu \mathrm{m}}^{13.0 \mu \mathrm{m}} \epsilon_{\lambda} E_{b \lambda}\left(T_{L}\right) d \lambda-W_{\mathrm{sur}}}{Q_{\mathrm{in}}} \cdot \chi_{\text {correction }}
$$

where $\epsilon_{\mathrm{Ir}}$ and $T_{\mathrm{IL}}$ are the thermal imager reference emissivity and the displayed infrared temperature, due to the correction factor, may be set to $1 ; \epsilon_{\mid r}$ is the index, determined by the thermal imager model, this experiment uses SAT-G95 thermal imager, index $n=4.09 ; \sigma$ is the Stefan-Boltzmann constant; $\epsilon_{\lambda}$ and is $T_{\perp}$ the emissivity of the Lambert target surface and its surface temperature, respectively, $\epsilon_{\lambda}=0.2, T_{\mathrm{L}}=320 \mathrm{~K} ; E_{b \lambda}$ is the spectral radiation force function; $Q_{\text {in }}$ is the real-time irradiance, obtained using a direct irradiance meter.

$W_{\text {sur }}$ includes the radiant energy of surrounding objects and the atmosphere:

$$
W_{\text {sur }}=\int_{7.5 \mu \mathrm{m}}^{13.0 \mu \mathrm{m}}\left[\epsilon_{\text {refl }} E_{b \lambda}\left(T_{\text {refl }}\right)+(1-\tau) E_{b \lambda}\left(T_{\text {atm }}\right)\right] \mathrm{d} \lambda
$$

where $\epsilon_{\text {refl }}$ and $T_{\text {refl }}$ are the emissivity and temperature of the surrounding objects, $\tau_{\text {atm }}$ and $T_{\text {atm }}$ are the transmittance and temperature of the surrounding atmosphere, respectively. According to the thermal imager help file, $\epsilon_{\text {refl }}=1.0, \tau_{\text {atm }}=0.92$.

$\chi_{\text {correction }}$ is obtained according to the results of flux distribution experiment and the relation of different infrared surface radiation:

$$
\chi_{\text {correction }}=\frac{Q_{\text {test,center }}}{\epsilon_{\mathrm{Ir}} \sigma T_{\mathrm{IL}, \text { center }}^{n}-\int_{7.5 \mu \mathrm{m}}^{13.0 \mathrm{~m}} \epsilon_{\lambda} E_{b \lambda}\left(T_{L}\right) d \lambda-W_{\text {sur }}}
$$

$Q_{\text {test,center }}$ is the central heat flux density of heat flow meter, $T_{\mathrm{IL}, \text { center }}$ is the central spot temperature of the infrared imager. 


\section{Results}

\subsection{Simulation}

At present, the commercial software in the field of optical engineering generally adopts the ray tracing method of sequence tracing [10]. This article implements the performance simulation of the CDTS system based on Fortrango, and simplifies the dish concentrator into a parabolic shape, using a square constraint. The results obtained by this method were compared with the results of MCRT method (Figure 5). Clear heat flux distribution image of surface was obtained with surface error 2.5 mrad, the specular reflection error is 2.5 mrad.

(a)

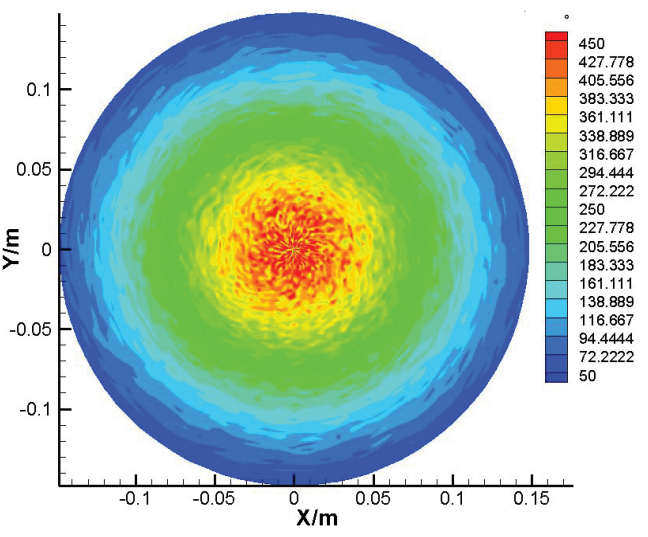

(b)

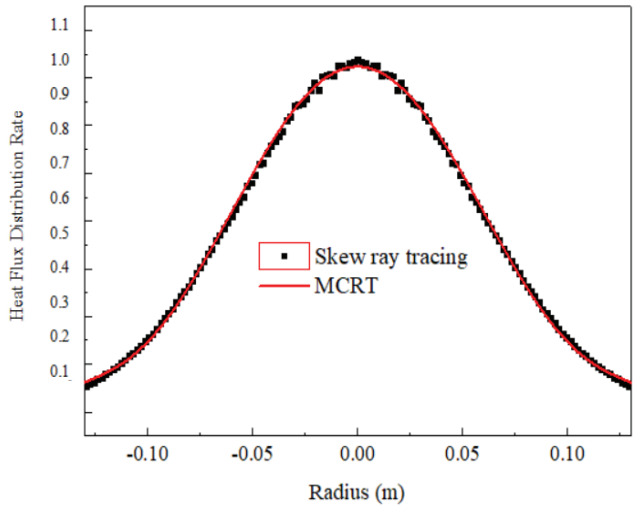

Figure 5: (a) Heat flux distribution, (b) comparison with MCRT simulation results.

The aggregated energy flow of the CDTS system exhibits a Gaussian distribution, changing sharply with radial direction. The peak energy flow density is about 450 $\mathrm{kW} / \mathrm{m}^{2}$, and most of the area of the concentrated spot is within $20 \mathrm{~cm}$. It can be seen that compared with the simulation results of MCRT method, it is found that the degree of coincidence is higher, indicating that the vector matrix modeling method adopted in this article has higher accuracy.

\subsection{Experimental results}

During the experimental process, the atmospheric environment (wind speed, atmospheric pollutants, and thin clouds) fluctuated, and the effective intercepting area of the heliostat and the effective reflection area of the concentrator also changed dynamically due to the change of the solar height angle and the tracking error. At the same time, due to air pollution, dust and other reasons, the surface was ash, and the reflectivity was seriously reduced. Because the experimental time was late this time, 
the solar altitude was low and blocked by some buildings, resulting in a low peak energy flow density at the center point.

According to the infrared thermal image analysis, the focal spot center temperature reached $570^{\circ} \mathrm{C}$, and the temperature gradually decreased as the spot radius increased. Through the thermal image diagram, the temperature distribution in the effective area of the spot can be obtained. Combined with the heat flux density of the central heat flow meter and the temperature value of the radial thermocouples, the spot heat flux density distribution and the heat flow distribution image along the radial direction were obtained (Figure 6). From the image, the experimental test shows that the effective diameter of the focal spot is about $20 \mathrm{~cm}$, and the peak energy density of the center is $400 \mathrm{~kW} / \mathrm{m}^{2}$. Due to the certain error in the field test, the shape of the spot is elliptical. In the range of $5 \mathrm{~cm}$ from the center point, the energy proportion value is $72 \%$.

(a)
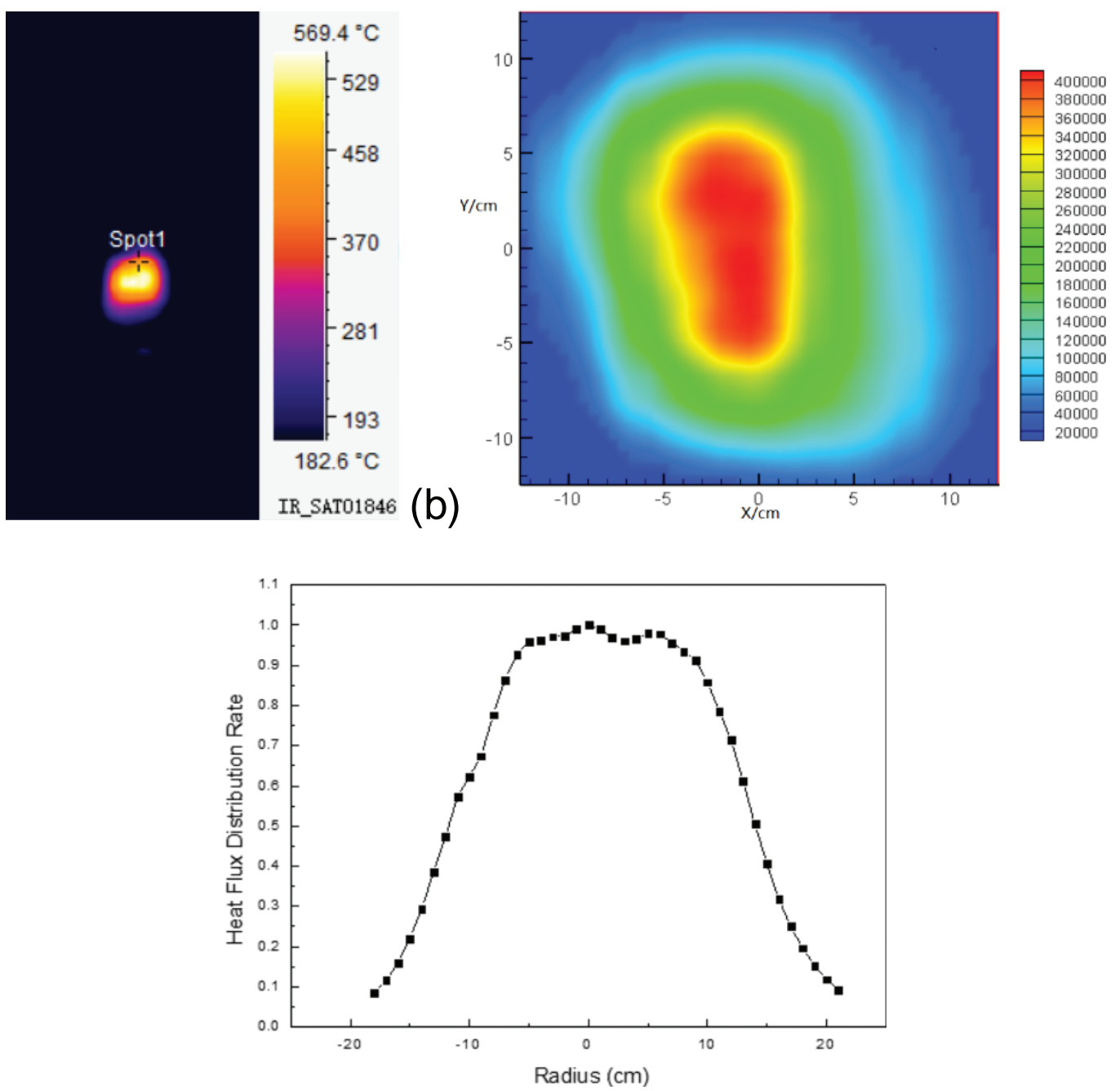

(c)

(b) 


\section{Conclusion}

In this experiment, the focal spot heat flux distribution of CDTS system was obtained based on the High-times Concentrating Directional Transmission System (CDTS) through this measurement. With the consideration of different errors and compared with Monte Carlo ray tracing method, the feasibility of the measurement system is verified. It is applicable to expand the range of space environments, while ensuring measurement accuracy, and has certain practical significance.

\section{References}

[1] Zhao, Y. W. (2003). The overview and tendency of solar energy utilization. China Electricity, vol. 36, no. 9, pp. 63-69.

[2] Ballestrin, J. (2002). A non-water-cooled heat flux measurement system under concentrated solar radiation conditions. Solar Energy, vol. 73, no. 73, pp. 159-168.

[3] Ballestrn, J., Ulmer, S., Morales, A., et al. (2003). Systematic error in the measurement of very high solar irradiance. Solar Energy Materials \& Solar Cells, vol. 8o, no. 3, pp. $375-381$.

[4] Ballestr, J. and Monterreal, R. (2004). Hybrid heat flux measurement system for solar central receiver evaluation. Energy, vol. 29, no. 56, pp. 915-921.

[5] Johnston, G. (1995). On the analysis of surface error distributions on concentrated solar collectors. Journal of Solar Energy Engineering, vol. 117, no. 4, pp. 294-296.

[6] Johnston, G. (1998). Focal region measurements of the $20 \mathrm{~m}^{2}$ tiled dish at the Australian National University. Solar Energy, vol. 63, no. 2, pp. 117-124.

[7] Lin, P. D. and Lu, C. H. (2004). Analysis and design of optical systems by use of sensitivity analysis of skew ray tracing. Applied Optics, vol. 43, pp. 796-807.

[8] Mousazadeh, H., Keyhani, A., Javadi, A., et al. (2009). A review of principle and suntracking methods for maximizing solar systems output. Renewable and Sustainable Energy Reviews, vol. 13, pp. 1800-1818.

[9] Cheng, K. and Zhang, H. (2006). Monte Carlo simulation of the solar radiation at the entrance surface of the collector. Acta Energiae Solaris Sinica, vol. 27, no. 8, pp. 743-747.

[10] Tsao, C. H., Freniere, E., and Smith, L. (2009). Improved predictive modeling of white LEDs with accurate luminescence simulation and practical inputs with TracePro opto-mechanical design software. Proceedings of SPIE. Light-Emitting 
Diodes: Materials, Devices, and Applications for Solid State Lighting XIII, p. 7231: UNSP 723111. 